\title{
Dual delivery of bone morphogenetic protein-2 and basic fibroblast growth factor from nanohydroxyapatite/collagen for bone tissue engineering
}

\author{
Yuqian $\mathrm{Hu}^{1,2,3}$, Linlin Zheng ${ }^{3}$, Jinhui Zhang ${ }^{3}$, Lijuan Lin ${ }^{3}$, Yue Shen ${ }^{3}$, Xiaoyan Zhang ${ }^{4^{*}}$ and Buling Wu $\mathrm{W}^{1,2^{*}}$
}

\begin{abstract}
Background: In bone tissue engineering, the fabrication and biocompatibility of scaffold are crucial. Among many scaffold materials, nanohydroxyapatite (nHAP) and collagen (COL) are chosen as building materials of scaffold. At the same time, growth factors were also used to modify the scaffolds.

Methods: In this study, blending and freeze drying methods were adopted together in order to build basic fibroblast growth factor (bFGF)-bone morphogenetic protein-2 (BMP-2)-nHAP/COL scaffolds. ELISA was applied to test the release of bFGF and BMP-2 on the scaffold. The flow cytometry was used to identify bone marrow mesenchymal stem cells (BMSCs). Scanning electron microscope was adopted to observe scaffolds and cells morphology. BMSCs were seeded on the scaffolds to test the biological compatibility in vitro. Cells were counted to detect early cell adhesion. Cell counting kit-8 assay was adopted to detect cell proliferation and alkalinephosphatase assay was applied to detect cell activity.

Results: The characterization of bFGF-BMP-2-nHAP/COL scaffolds meets the requirements of ideal bone tissue engineering scaffolds. BMSCs that were isolated, purified and passaged satisfied the needs of further experiments. The growth status of cells on bFGF-BMP-2-nHAP/COL scaffolds was satisfactory. Cell adhesion was the highest in the bFGF-BMP-2-nHAP/COL scaffolds group. The cell viability and ALP activity of bFGF-BMP-2-nHAP/COL scaffolds group were the highest.
\end{abstract}

Conclusion: Taken together, bFGF-BMP-2-nHAP/COL scaffolds have good biocompatibility in vitro and promote adhesion, proliferation, differentiation of BMSCs.

Keywords: Bone tissue engineering, Nanohydroxyapatite, Collagen, BMP-2, bFGF

\section{Introduction}

In the past decade, tissue engineering has been playing increasingly important role in orthopaedic repair. Tissue engineering combined cells and its growth factors with biomaterials through its own principles, has been already adopted to develop various of connective

\footnotetext{
*Correspondence: 15941587899@163.com; bulingwu@smu.edu.cn ${ }^{1}$ Department of Stomatology of Nanfang Hospital, Southern Medical University, Guangzhou 510000, China

${ }^{4}$ Department of Pathology, Fifth People's Hospital of Shenyang City \&

Shenyang Tumor Hospital, Shenyang 110023, China

Full list of author information is available at the end of the article
}

tissues, in vivo and vitro, such as cartilage, bone, tendon or ligament [1]. The interest in bone tissue engineering is constantly growing in parallel with the rise in trauma victims and musculoskeletal disorders associated with the increase in life expectancy [2,3]. To develop an artificial bone matrix, a tissue engineering scaffold always works as a pivotal element in regenerating bone tissue [4]. The construction of scaffolds requires the combination of a variety of materials, and the methods of construction are varied. In this experiment, nanohydroxyapatite (nHAP) and collagen (COL) were selected as the basic components, bone morphogenetic protein 2 (BMP-2) and basic 
fibroblast growth factor (bFGF) were combined with them by mechanical mixing and freeze drying methods to form a double factor scaffold (bFGF-BMP-2-nHAP/ COL). BMP-2 in $100 \mathrm{ng} / \mathrm{mL}$ and bFGF in $50 \mathrm{ng} / \mathrm{mL}$ both are beneficial to promote the proliferation of bone marrow mesenchymal stem cells (BMSCs). Furthermore, the combined utilization of BMP-2 and bFGF at a ratio of 2:1, significantly promotes the proliferation and differentiation of BMSCs compared with BMP-2 or bFGF use alone [5]. Su et al. had also demonstrated the feasibility of using double factor release in poly(lactic-co-glycolic acid)/ polycaprolactone/nanohydroxyapatite (PLGA/PCL/ nHAP) scaffold [6]. The nHAP have been increasingly used in biomaterial composites because of their excellent biocompatibility, bioactivity, osteoconductivity, and osseointegrative nature [7-11]. Several research revealed that COL improves cell adhesion, promotes bone cell proliferation and enhances osteogenic cell differentiation [12, 13]. Moreover, the initial adhesion of periosteum segments was greatly improved by $\mathrm{COL}$, which thus facilitated cell outgrowth and handling efficiency on implantation $[14,15]$. The bFGF regulates a variety of biological functions including neuroprotection, vasodilation, the stimulation of angiogenesis and the suppression of apoptosis [16-18]. Among the bone morphogenetic proteins (BMPs), BMP-2 has been profoundly studied in osteogenesis and exerts superior potential in bone formation [19-21]. In this study, the morphology of the dual factor scaffold and its protection and growth-promoting effects on BMSCs were tested to see if the requirements for bone tissue engineering were met.

\section{Materials and methods Materials}

Rat tail tendon collagen Typelwas purchased from Shengyou Biotechnology (Hangzhou, Zhejiang, China). The nHAP was purchased from Emperor Nano Material (Nanjing, Jiangsu, China). Acetic acid, anhydrous ethanol, HCL, cell counting kit-8 (CCK-8), alkalinephosphatase (ALP) kit was purchased from Nanjing Jiancheng Bioengineering Institute. Phosphate buffer solution (PBS), $\alpha M E M, 10 \%$ fetal bovine serum (FBS), $0.25 \%$ trypsin was purchased from GE Healthcare Life Sciences Hyclone Labratry (South Logan, Utah, USA). The bFGF was purchased from PeproTech (catalog Number: 40029, Rochy Hill, USA). BMP-2 was purchased from PeproTech (catalog Number: 120-02, Rochy Hill, USA). TrypLE Express was purchased from Gibco (USA). FITC anti-rat CD44H, APC anti-mouse/rat CD29, PE anti-rat CD45 were purchased from Biolegend, Inc. (CA, USA). CD34 Polyclonal antibody PE conjugated was purchased from AOsen Biotechnology (Beijing, China). All other general laboratory reagents were of the highest quality available and obtained through standard commercial suppliers.

\section{Animals}

4-week-old SD rats were acquired from the Center for Experimental Animals at Medical College of Eastern Liaoning University (Dandong, China) with a National Animal Use License number of SCXK-LN2011-0009 and allowed a 1-week adaptation before experiments began. Food and water were provided ad libitum. The rats were cultivated at a constant temperature $\left(25 \pm 1{ }^{\circ} \mathrm{C}\right)$, humidity $(60 \pm 5 \%)$ and 12-h light/dark cycles (6 a.m. to 6 p.m.).

\section{Preparation of bFGF-BMP-2-nHAP/COL scaffolds}

$10 \mathrm{mg}$ COL was added into $10 \mathrm{~mL}$ acetic acid solution $(0.005 \mathrm{~mol} / \mathrm{L})$ and intensively mixed by stirring with a magnetic stir bar. $10 \mathrm{mg}$ nHAP was then added to the above solution and stirred overnight. The quality ratio of the above method (nHAP: COL) was 1:1.5. $5 \mathrm{ng} / \mu \mathrm{L}$ bFGF and $10 \mathrm{ng} / \mu \mathrm{L}$ BMP-2 solutions were prepared by dissolving standards in PeproTech protein solution [22] and then added to the solution of the scaffold and stirred at low temperature for $50 \mathrm{~min}$, so that the final concentration of bFGF and BMP-2 in the mixed solution was $50 \mathrm{ng} / \mathrm{mL}$ and $100 \mathrm{ng} / \mathrm{mL}$. The solution was then subjected into a 24-hole Teflon culture plate, storing at $-20{ }^{\circ} \mathrm{C}$ for $24 \mathrm{~h}$ and then lyophilized at $-80^{\circ} \mathrm{C}$ for $48 \mathrm{~h}$ to obtain bFGFBMP-2-nHAP/COL scaffolds. According to this method, the bFGF-nHAP/COL, BMP-2-nHAP/COL, nHAP/COL scaffolds were prepared. The morphology and microstructure of the scaffolds was observed by scanning electron microscope (SEM) (Hitachi-4800, Tokyo, Japan).

\section{Detection of $\mathrm{bFGF}$ and BMP-2 release \\ in bFGF-BMP-2-nHAP/COL scaffolds}

After $1 \mathrm{~mL}$ bFGF-BMP-2-nHAP/COL solution was fabricated to form one standard scaffold (bFGF $50 \mathrm{ng}$, BMP-2 $100 \mathrm{ng}$ ), three scaffolds were placed into the orifice plate and $1 \mathrm{~mL} 1 \times$ PBS buffer ( $\mathrm{PH}=7.4)$ was then added into the orifices, following which were sealed by the membrane. The orifice plate was placed in the cell culture box at $37^{\circ} \mathrm{C}$, and PBS buffer in pores were collected, at specific time points (1, 2 to 20 days). After collecting liquid, $1 \mathrm{~mL}$ PBS buffer was added into the hole and then bFGF and BMP-2 ELISA kit (Yuduo Biotechnology company, Shanghai, China) was used to detect the bFGF and BMP-2 content of each sample, following which took the mean and calculated the cumulative release of bFGF and BMP-2, and drew the curve [6]. According to the above method, bFGF of bFGF-nHAP/COL and BMP-2 of BMP2-nHAP/COL were detected. 


\section{Isolation, culture and passage of BMSCs}

4 week old SD rat was sacrificed by excess of anesthesia and $75 \%$ ethanol was applied to sterilize the skin. Under aseptic conditions, the femur and tibia were removed and soaked in PBS solution. After ultraviolet disinfection, PBS containing penicillin and streptomycin was adopted to wash bone for three times. After the bone marrow cavity was exposed, the $5.2 \mathrm{~mL}$ cell growth fluid was drawn into until most of the bone marrow was flushed out. After being blown and sucked three times, the cell suspension was sucked into a sterile container. After counting, the cells were inoculated in the culture dish according to the required concentration. The $\alpha$ MEM medium containing $10 \%$ fetal bovine serum and $1 \%$ penicillin and streptomycin was added into the culture medium, the cells were cultured at $37{ }^{\circ} \mathrm{C}$, in a $\mathrm{CO}_{2}$ incubator, and changed liquid every $24 \mathrm{~h}$. After BMSCs adhering the wall deformated and gathered, the cells were digested using TrypLe Express enzyme 2.5-3.0 mL for $10 \mathrm{~min}$ at $37^{\circ} \mathrm{C}$ until $\alpha \mathrm{MEM}$ was used to terminate the reaction. At the speed of $800 \mathrm{rpm}$, the cell suspension was centrifuged for $5 \mathrm{~min}$ and the cells were resuspended in an aMEM medium and seeded into a new $10 \mathrm{~cm}$ (diameter) culture dish. Cells were changed liquid one time every day, and then was 1:3 passaged after being fused to the $80-90 \%$.

\section{BMSCs identification}

The third generation of BMSCs was used for identification. After discarding the medium, the cells were washed by PBS. TrypLE Express was added into and the cells were digested at $37^{\circ} \mathrm{C}$ for 10 min until the $\alpha M E M$ medium was used for the termination of the reaction. After the cell suspension was charged, the cells were counted after centrifugation and being adjusted to $10^{6} / 100 \mu \mathrm{L}$ and then PBS containing $10 \% \mathrm{BSA}(\mathrm{w} / \mathrm{v})$ was added to close. The cell suspension was transferred to 4 round bottom flcon tubes and the APC labeled anti-CD29 antibody, FITC labeled anti-CD44 antibody, PE labeled anti-CD29 antibody, PE labeled anti-CD34 antibody were added into the cell suspensions respectively according to the final concentration of $0.2 \mathrm{~g} / 100 \mathrm{~mL}$. After being incubating at $4{ }^{\circ} \mathrm{C}$ for $20 \mathrm{~min}$, the surface markers CD34, CD29, CD44 and CD45 were analyzed by the flow cytometry (FACSCanto II, BD Company, USA) [23].

\section{BMSCs culture}

After the lyophilized bFGF-BMP-2-nHAP/COL scaffolds were sterilized with ethylene oxide (SQ-H, Zhongxitai Service, Beijing, China), the scaffolds was washed with PBS and $\alpha M E M$ medium three times, and then soaked into FBS overnight. The scaffolds were placed in 24 hole cell culture plate, and the concentration of the BMSCs suspension was adjusted to $2 \times 10^{5} / \mathrm{mL}$. After each scaffold was inoculated with $1 \mathrm{~mL}$ suspension for $24 \mathrm{~h}, 1 \mathrm{~mL}$ culture medium replaced the cell suspension and was added into the hole around the scaffold and then the cell scaffold was cultured in $5 \% \mathrm{CO}_{2}$ incubator at $37{ }^{\circ} \mathrm{C}$ [24]. Six independent experiments were conducted.

\section{BMSCs morphology}

After the cells were inoculated for $72 \mathrm{~h}$, the samples were taken out, and washed with PBS, and then added to glutaraldehyde solution, which was then placed in a wet box and fixed overnight at $4{ }^{\circ} \mathrm{C}$. The samples were removed and washed by PBS, dehydrated with ethanol gradient (50\%, $70 \%, 80 \%, 90 \%$, and $100 \%$, each lasting for about $20 \mathrm{~min}$ ), and then dried by natural drying method and sprayed with gold. Finally, SEM was used to observe the samples.

\section{Adhesion of BMSCs}

bFGF-BMP-2-nHAP/COL, bFGF-nHAP/COL, BMP-2nHAP/COL, nHAP/COL and control groups were set up. The BMSC cells at the density of $1 \times 10^{4} / \mathrm{mL}$ were placed in culture plates, which were pre-coated with the bFGFBMP-2-nHAP/COL, bFGF-nHAP/COL, BMP-2-nHAP/ COL, nHAP/COL scaffolds (1 mL per well) and BMSCs that were cultured in plates with no scaffold worked as control. A total of six parallel wells were used for each group and the cells were cultured at a $37{ }^{\circ} \mathrm{C}$ humidified incubator with $5 \% \mathrm{CO}_{2}$. The amounts of non-adhered cells were quantified at $1,2,4,8$ and $24 \mathrm{~h}$ and the adhesion rate was calculated according to the following formula: Adhesion rate $(\%)=($ number of seeded cells - non-adhered cells) /(number of seeded cells) $\times 100$ [25].

\section{CCK-8 assay}

CCK- 8 kit was used to detect the proliferation of cells in four scaffold groups and control group. At 1, 3, 5, and $7 \mathrm{~d}$ after inoculation, 3 samples were taken out. $100 \mu \mathrm{L}$ CCK-8 solution was added into each hole. $300 \mu \mathrm{L}$ solutions from each hole was added to the 96 hole culture plate and the absorbance value of the solution was detected by the microplate reader $(\lambda=450 \mathrm{~nm})$ (Bio-Rad 550 Bio-Rad Laboratories, Inc., $\mathrm{CA}$, USA). The average value of 3 parallel samples was taken as the experimental data. Optical density (OD) at $450 \mathrm{~nm}$ was applied to represent the number of cells [26].

\section{ALP activity}

At 1, 4, 7 and 10 days after culture, 3 samples were taken out from each group, respectively, and then washed with PBS 3 times. $1 \mathrm{~mL}$ 0.1\% TritonX-100 was added to decompose cells at $4{ }^{\circ} \mathrm{C}$ for overnight aiming. Straw was used to decompose the cells completely and then $30 \mu \mathrm{L}$ suspension was transferred into the 96 orifice plate. $50 \mu \mathrm{L}$ buffer solution and matrix liquid were configured, and fully mixed following water bath for $15 \mathrm{~min}$. After that, each hole was added into $150 \mu \mathrm{L}$ chromogenic agent and oscillated, and the OD of $520 \mathrm{~nm}$ was measured by ELISA [27]. 


\section{Ethics}

All animal studies were performed after receiving the approval of the Animal Use and Care Committee at Medical College of Eastern Liaoning University with a protocol number EMU62043006. All experiments were approved by the Animal Care and Use Committee at Medical College of Eastern Liaoning University, and complied with the National Institutes of Health Guide for the Care and Use of Laboratory Animals.

\section{Statistical analysis}

All data are presented as the mean \pm standard deviation (SD). Statistical analyses were performed by SPSS17.0 (SPSS, Inc., IL, USA). Multiple groups comparison was analyzed by one-way analysis of variance (ANOVA) and Student's t-test was adopted to comparison between two groups. $P<0.05$ was regard as statistically significant. Prior to results being analyzed using one-way ANOVA, all quantitative data were confirmed as normally distributed.
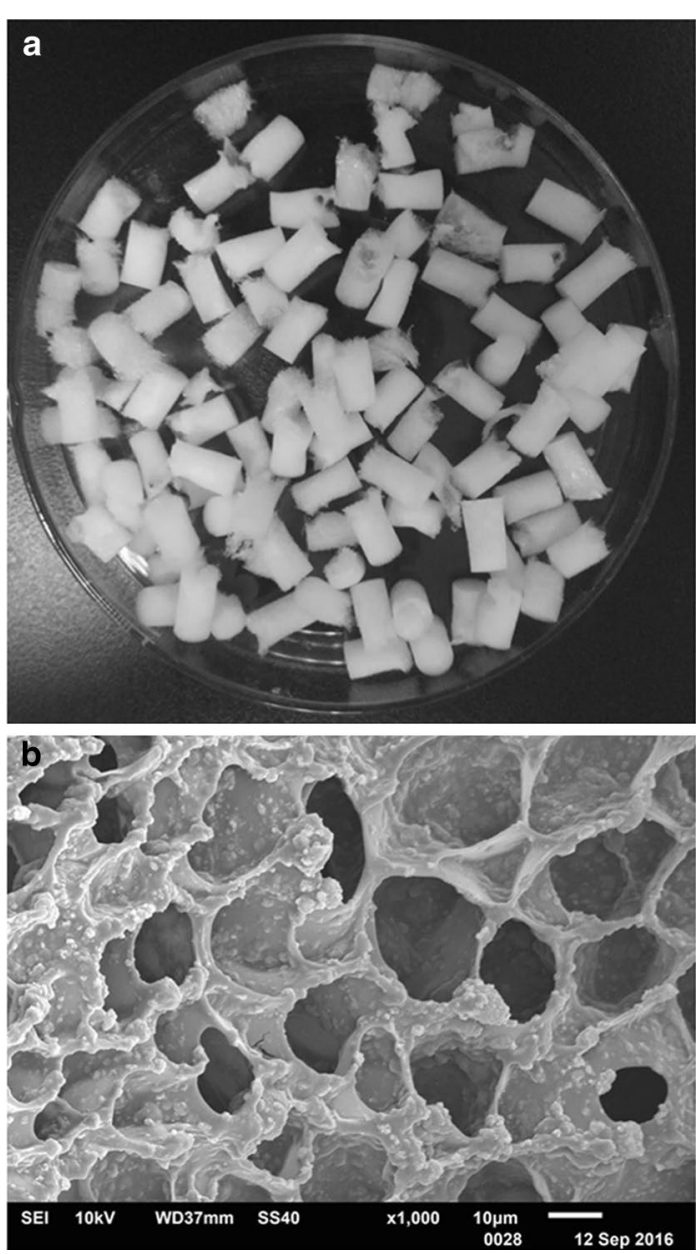

Fig. 1 a Gross morphology of the bFGF-BMP-2-nHAP/COL scaffolds. b SEM images of the bFGF-BMP-2-nHAP/COL scaffold (magnification $\times 1000)$
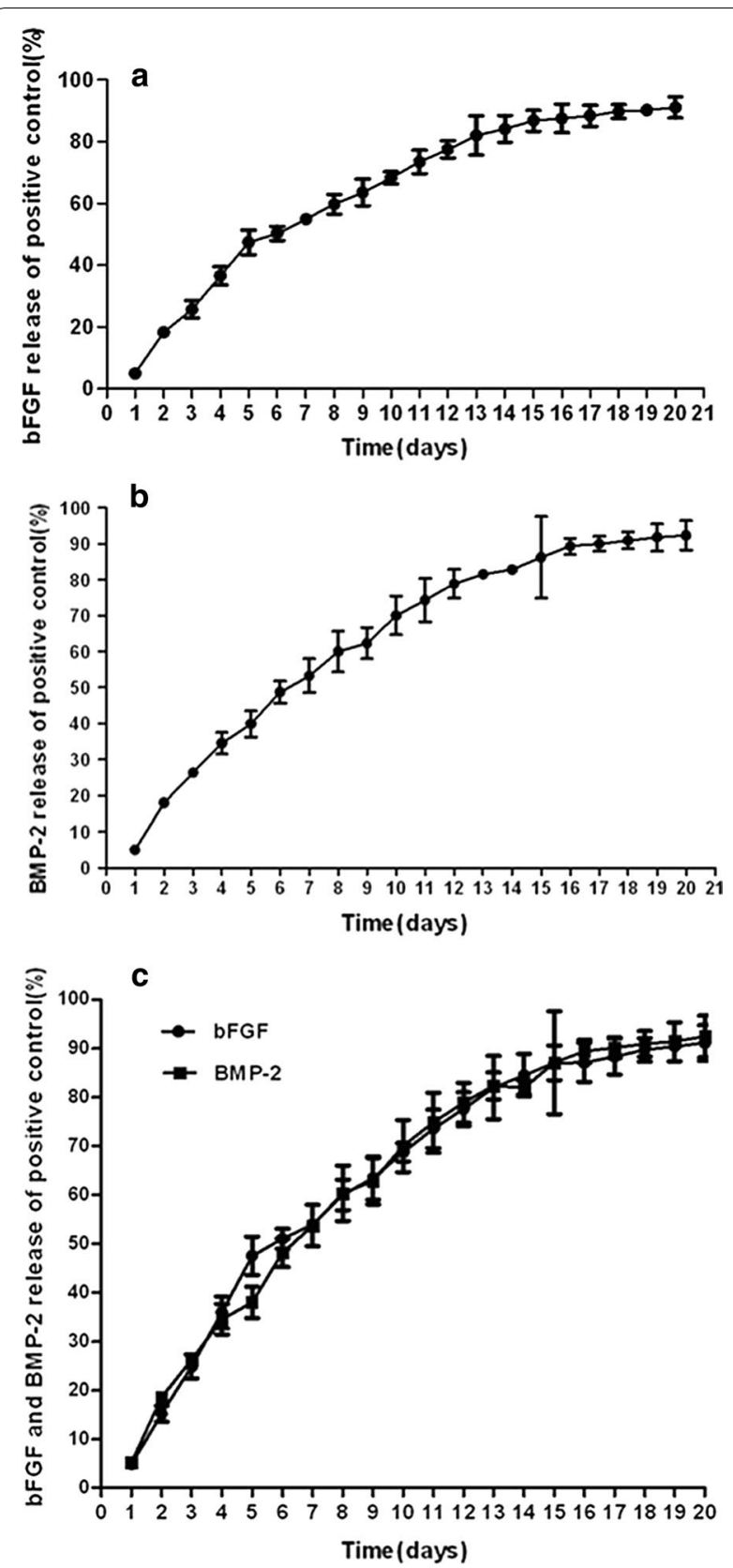

Fig. 2 a Cumulative release rate of bFGF from bFGF-nHAPCOL scaffolds. $\mathbf{b}$ Cumulative release rate of BMP-2 from BMP-2-nHAP/COL scaffolds. c Cumulative release rate of bFGF and BMP-2 per time point from bFGF-BMP-2-nHAP/COL scaffolds

\section{Results}

Morphology and SEM image of bFGF-BMP-2-nHAP/COL scaffold

Scaffolds was white, the flexibility was good and the compression deformation could be gradually restored to the original state, the surface was slightly rough (Fig. 1a). The sample was three-dimensional porous structure and a large numbers of nHAP particulates could be seen to 

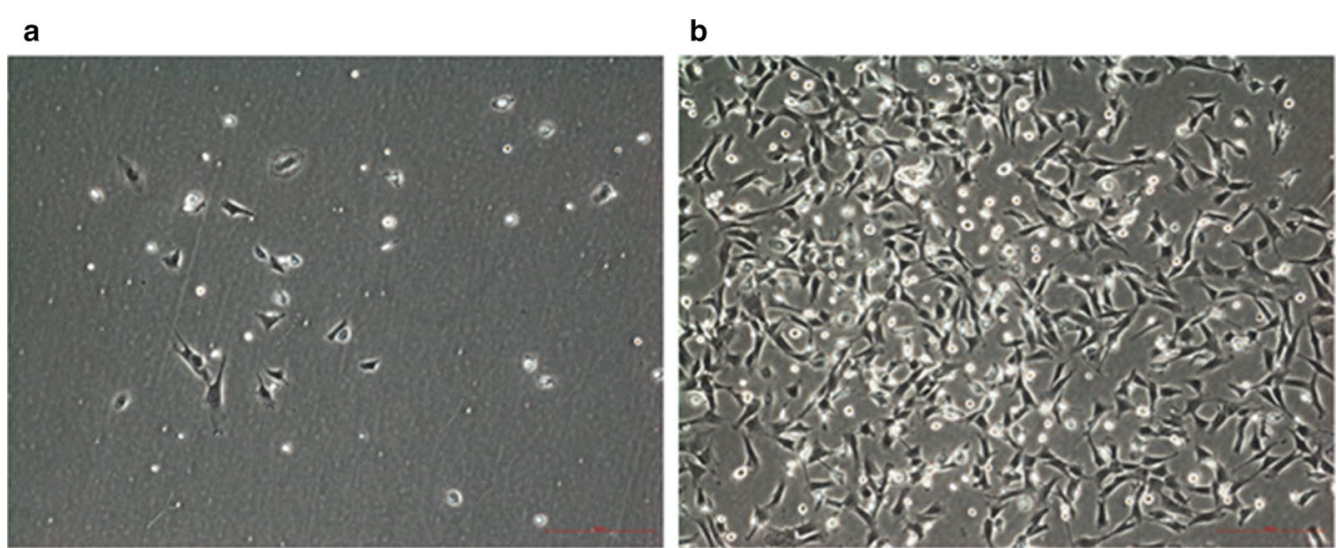

c

d
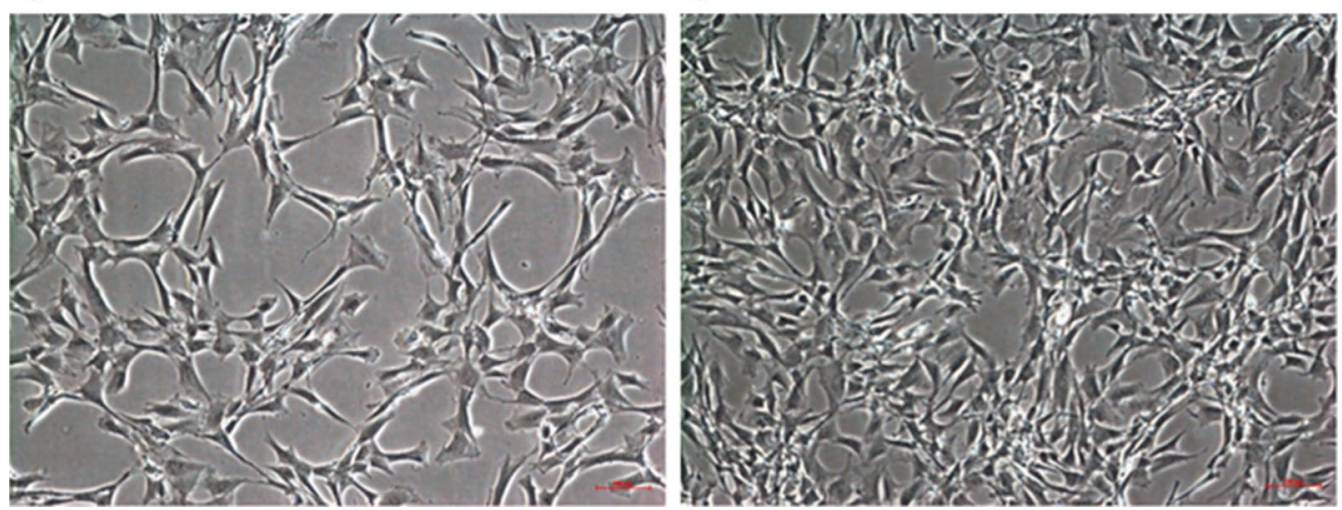

Fig. 3 Cell morphology of BMSCs, examined under a light-inverted microscope (magnification $\times 100$ ). a 1 day after primary BMSCs were cultured. $\mathbf{b}$ 5 days after primary BMSCs were cultured. $\mathbf{c} 1$ day after passage 1 BMSCs were cultured. $\mathbf{d} 5$ days after passage 1 BMSCs were cultured

adhere on the surface COL. The aperture was between 80 and $200 \mu \mathrm{m}$ and the holes were connected with each other, there was no obvious fixed direction. The thickness of the hole wall was about $1-2 \mu \mathrm{m}$ and the combination of nHAP and COL was homogeneous and substantial (Fig. 1b).

\section{Release of bFGF and BMP-2 in scaffolds}

$b F G F$ in bFGF-nHAP/COL scaffolds could be accumulated to release for 17 days. After 18 days, there was only little amount of bFGF factor $(<1 \mathrm{ng})$ in the supernatant of each sample or not be detected at all. The amount of factor released by the scaffold material was $91.05 \pm 3.38 \%$ of that of factor added. In 3-5 days, the burst release of factor appeared in the scaffolds, and the release tended to be stable after 5 days (Fig. 2a). BMP-2 in BMP-2nHAP/COL scaffolds could be accumulated to release for 19 days. The amount of factor released was $90.05 \pm 2.08 \%$. In 2-5 days, the burst release of factor appeared, and the release tended to be stable after 5 days (Fig. 2b). The release of bFGF and BMP in double factor scaffolds was not significantly different from that of the single factor scaffold according to the curve (Fig. 2c).

\section{Cell morphology}

Primary BMSCs were inoculated for $12 \mathrm{~h}$ and then gradually began to adhere to the wall. After 1 day, some cells began to deform, and cells showed Spindle or polygonal, nucleus was large and deeply stained. Some adherent cells are round or oval, and nucleus was large and deeply stained, at the same time, non adherent red blood cells can be seen (Fig. 3a). 1 day after the first vaccination, non adherent cells and the cells that need a longer time to adhere to the wall were cleared. About 5 days after the culture, BMSCs were collected and a small number of round cells were mixed in spindle and polygonal cells (Fig. 3b). After digestion and re-inoculation using TrypLE Express, the number of impurity cells decreased significantly and at 6 days, the cells adhered the wall and deformed. After the first passage, BMSCs grew well, and the impurity cells were significantly reduced (Fig. 3c). 3 days after the second inoculation, cell density increased and at about 9 days, BMSCs completed the fusion of $80 \%$ to $90 \%$ and could be passaged (Fig. 3d). 

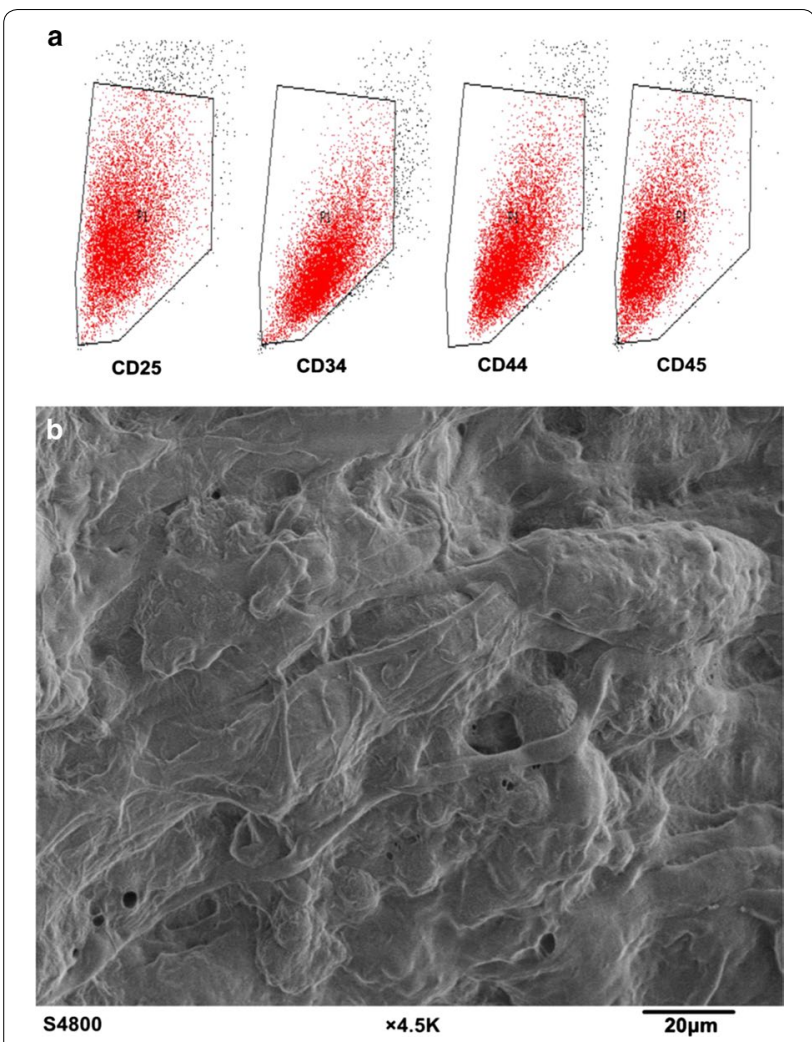

Fig. 4 a Flow cytometry analysis of BMSCs. b Scanning electron microscopes images of BMSCs that were inoculated on the bFGF-BMP-2-nHAP/COL scaffold after 7 days

\section{BMSCs identification}

The results of flow cytometry of the third generation BMSCs antibody markers were as follows: the positive rates of CD45, CD34, CD44, CD29 were 4.4\%, 6.8\%, 94\%, $100 \%$ respectively. The above results were consistent with the standard of BMSCs flow cytometry (CD45 and CD34 positive rate of $<10 \%, C D 44$ and CD29 positive rate of $>90 \%$ ) (Fig. 4a).

\section{BMSCs cultured with the bFGF-BMP-2-nHAP/COL scaffold indicate biocompatibility}

The SEM images showed randomly distributed cells on the surface. After 7 days, an increased number of cells had adhered to the surface of the scaffold, the cell had grown and proliferated well. The cells exhibited a typical BMSCs morphology, and adhered tightly on the scaffold surface via the formation of lamellipodia and filopodia, indicative of cell spreading (Fig. 4b).

\section{Dual delivery of bFGF and BMP-2 promote the adhesion of BMSCs}

The cell adhesion rate was enhanced with increasing incubation period in five groups (Fig. 5a). Following culture for 1,3 , and $6 \mathrm{~h}$, the adhesion rate of cells in groups pretreated with scaffolds was higher than that of the control group $(\mathrm{P}<0.05)$. The adhesion rate of bFGFBMP-2-nHAP/COL, bFGF-nHAP/COL and BMP-2nHAP/COL groups was higher compared to that of nHAP/COL group $(\mathrm{P}<0.05)$, and bFGF-BMP-2-nHAP/ COL group with two factors was higher than above single factor groups. The adhesion rates of BMP-2-nHAP/ COL group and bFGF-nHAP/COL group were not significantly different $(\mathrm{P}>0.05)$. These results demonstrated that bFGF-BMP-2-nHAP/COL scaffold increased the adhesion of BMSCs better (Fig. 5a).

\section{Dual delivery of bFGF and BMP-2 promote the proliferation of BMSCs}

The proliferation of BMSCs in scaffold groups and the control group cultured for 1, 3, 5, 7 days were compared using a CCK-8 assay. The absorbance values for the scaffolds increased indicating significant cell growth within the scaffolds. The number of cells increased with culture duration in five groups. BMSCs proliferation was observed in five groups, and there was no significant difference between the scaffold groups and the control group after 1 day $(\mathrm{P}>0.05)$. However, after 3 days, the number of cells in the scaffold groups was higher than that in the control group $(\mathrm{P}<0.05)$. bFGF-BMP-2nHAP/COL, bFGF-nHAP/COL was higher than nHAP/ COL, BMP-2-nHAP/COL groups $(\mathrm{P}<0.05)$. There was no significant difference between BMP-2-nHAP/COL and nHAP/COL, bFGF-BMP-2-nHAP/COL and bFGFnHAP/COL $(\mathrm{P}>0.05)$. These results demonstrated that bFGF-BMP-2-nHAP/COL increase the proliferation of BMSCs (Fig. 5b).

\section{Dual delivery of bFGF and BMP-2 promote the differentiation of BMSCs}

The ALP activity of BMSCs cultured in scaffold groups and the control group was shown in Fig. 5c. No significant difference in OD values were identified between the scaffold groups and the control group in the first 4 days $(\mathrm{P}>0.05)$. However, The OD value in the scaffold groups was significantly higher than the control group between 4 and 10 days $(\mathrm{P}<0.05)$. Comparing factor groups with nHAP/COL group, the former was higher than the latter $(\mathrm{P}<0.05)$. This suggested that the nHAP/ COL scaffold had an effective impact on the activity of the BMSCs and bFGF, BMP-2 enhanced the impact further. In addition, dual factors group was higher than single factor groups $(\mathrm{P}<0.05)$, which suggested that bFGF-BMP-2-nHAP/COL promotes the differentiation of BMSCs (Fig. 5c). 

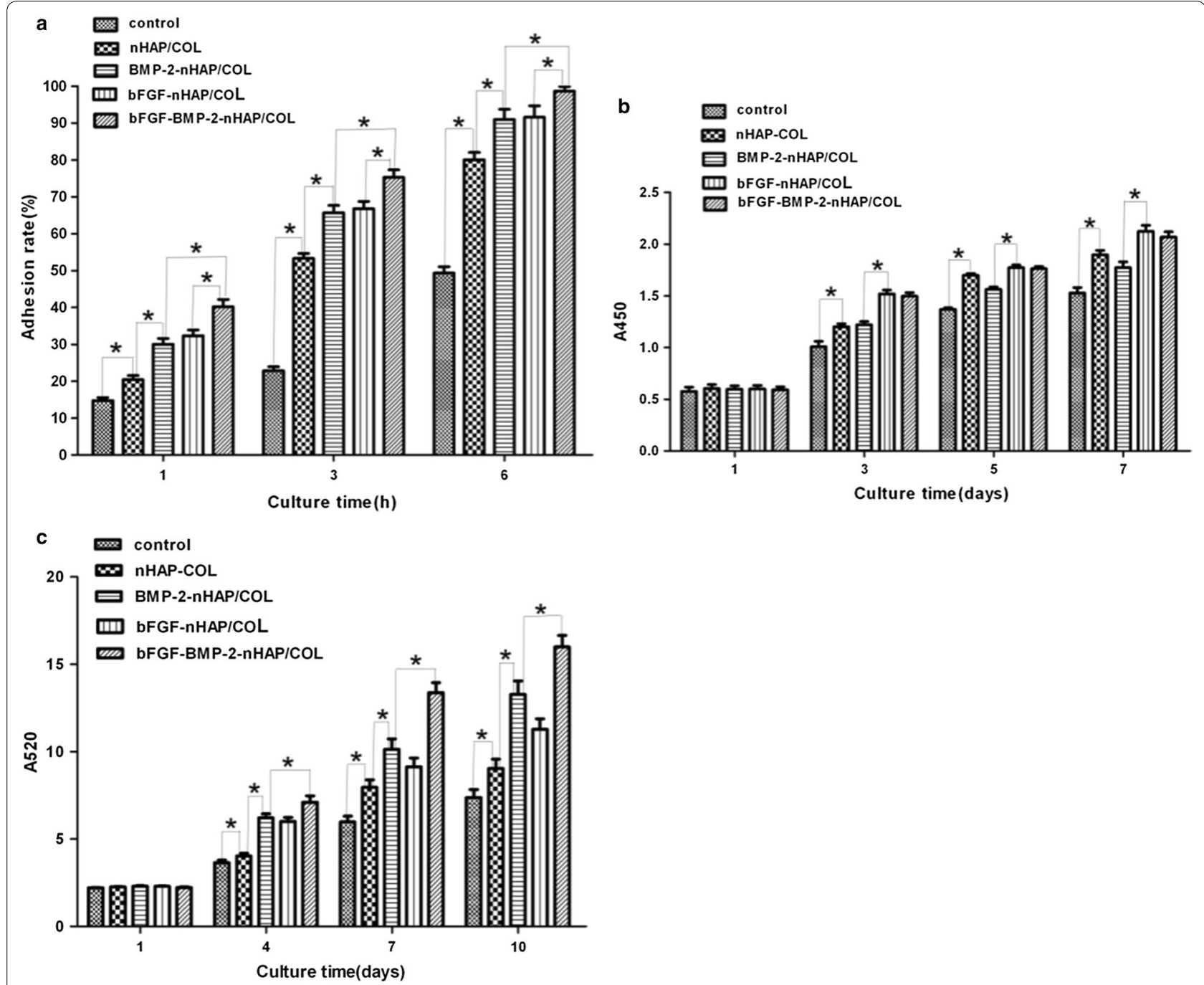

Fig. 5 a Adhesion rate of BMSCs cultured with the control, nHAP/COL, BMP-2-nHAP/COL, bFGF-nHAP/COL and bFGF-BMP-2-nHAP/COL groups. Data are expressed as the mean \pm standard deviation. ${ }^{*} A$ value of $P<0.05$ were considered statistically significant. $\mathbf{b}$ Cell counting kit- 8 assay determination of the proliferation of BMSCs from the control, nHAP/COL, BMP-2-nHAP/COL, bFGF-nHAP/COL and bFGF-BMP-2-nHAP/COL groups after 3 days. Data are expressed as the mean \pm standard deviation. ${ }^{*}$ A value of $P<0.05$ were considered statistically significant. c Alkaline phosphatase assay of the proliferation of BMSCs cultured with the control, nHAP/COL, BMP-2-nHAP/COL, bFGF-nHAP/COL and bFGF-BMP-2-nHAP/ $\mathrm{COL}$ groups. Data are expressed as the mean \pm standard deviation. ${ }^{*} \mathrm{~A}$ value of $P<0.05$ were considered statistically significant

\section{Discussion}

Collagen, an important extracellular matrix (ECM), has been extensively applied to facilitate cell growth and differentiation in tissue remodelling. The bio-inductive, desirable mechanical and degradable characteristics makes collagen a popular clinical application material [27]. After repeated lyophilization, the antigen of the collagen extracted of the rat tail was reduced further [28]. Hydroxyapatite, a biocompatible material with osteoconductive properties, can be available in various forms according to its applications, such as the bone formation and graft incorporation. Unfortunately, the disadvantages of bone-graft substitutes consisting solely of particles are mechanical weak and the migration from the graft site before ingrowth of new bone tissue secures them in place [29]. Due to its desirable biological and biomechanical properties, nano-scale biomaterials have drawn the attention of the research community [30]. The nHAP-COL scaffold provides a good spongy porous structure that satisfy the ideal scaffold material that provide a three-dimensional space for the transfer of nutrients of bone cells [31]. At the same time, the nHAP particles increased the roughness of the surface, thereby increasing the surface area of the 
scaffold, which is more conducive to cell adhesion. The release rate of bFGF and BMP-2 combined with scaffolds after lyophilization showed that the method had no significant loss in the process of production, and the sustained release time after lyophilization could meet the needs of tissue engineering. The results of flow cytometry showed that the isolated and purified cells were able to meet the requirements of further experiments. SEM images confirmed the good growth state of BMSCs on the scaffolds. The determination of cell adhesion rate confirmed the promoting effect of the composite scaffold and the role of bFGF and BMP-2 in promoting adhesion. The CCK- 8 method proved the effect of the compound scaffold on promoting proliferation. However, at the concentration of $10 \mathrm{ng} / \mu \mathrm{L}$, BMP-2 does not have a further role in promoting proliferation. The ALP method confirmed the good effect of the compound scaffold to promote differentiation and the obvious function of promoting differentiation of bFGF and BMP-2.

In this study, we revealed that bFGF-BMP-2-nHAP/ COL, two factors composite scaffold, can better promotes BMSCs adhesion, proliferation and differentiation, and be better applied to the further study for bone tissue engineering.

\begin{abstract}
Abbreviations
BMP-2: bone morphogenetic protein-2; bFGF: basic fibroblast growth factor; nHAP: nanometer hydroxyapatite; COL: collagen; ELISA: enzyme linked immunosorbent assay; BMSCs: bone marrow mesenchymal stem cells; SEM: scanning electron microscope; CCK-8: cell counting kit-8; ALP: alkalinephosphatase; PLGA: poly(lactic-co-glycolic acid); PCL: polycaprolactone; PBS: phosphate buffer solution; FBS: fetal bovine serum; OD: optical density; ANOVA: analysis of variance.
\end{abstract}

\section{Acknowledgements}

Not applicable.

\section{Authors' contributions \\ LL, WB and HY designed the study; HY, ZJ, and ZL performed the experi- ments; SY, and ZY analyzed the data; ZX and LL collected the clinical samples; $\mathrm{HY}$ and ZX drafted the manuscript. All authors read and approved the final manuscript.}

\section{Funding}

This work is supported by the Liaoning Science \& Technology Project (20170540404).

\section{Availability of data and materials}

The datasets used and/or analyzed during the current study are available from the corresponding author on reasonable request.

\section{Ethics approval and consent to participate}

In the present study, 4 week old SD rat was obtained from the Center for Experimental Animals at Medical College of Eastern Liaoning University (Dandong, China) with a National Animal Use License number of SCXKLN2011-0009 and executed in excess of anesthesia. Animal use was approved by the Animal Use and Care Committee at Medical College of Eastern Liaoning University with a protocol number EMU62043006. All experiments were approved by the Animal Care and Use Committee at Medical College of Eastern Liaoning University, and complied with the National Institutes of Health
Guide for the Care and Use of Laboratory Animals. All efforts were made to minimize the number of animals used and their suffering.

\section{Competing interests}

The authors declare that they have no competing interests.

\section{Author details \\ ${ }^{1}$ Department of Stomatology of Nanfang Hospital, Southern Medical University, Guangzhou 510000, China. ${ }^{2}$ Department of Stomatology, College of Stomatology, Southern Medical University, Guangzhou 510515, Guang- dong, China. ${ }^{3}$ Institute of Molecular Medicine, Medical College of Eastern Liaoning University, Dandong 118000, China. ${ }^{4}$ Department of Pathology, Fifth People's Hospital of Shenyang City \& Shenyang Tumor Hospital, Shen- yang 110023 , China.}

Received: 4 May 2019 Accepted: 13 August 2019

Published online: 27 September 2019

\section{References}

1. Helen HL, Subramony DS, Boushell MK, Zhang XZ (2010) Tissue engineering strategies for the regeneration of orthopaedic interfaces. Ann Biomed Eng 38:2142-2154

2. Wozney JM, Seeherman HJ (2004) Protein-based tissue engineering in bone and cartilage repair. Curr Opin Biotechnol 15:392

3. Cattalini JP, Pharm M, Boccaccini AR, Habil D, Lucangioli S, Mouriño V (2012) Bisphosphonate-based strategies for bone tissue engineering and orthopedic implants. Tissue Eng Part B Rev. 18:323-340

4. Thavornyutikarn B, Chantarapanich N, Sitthiseripratip K, Thouas GA, Chen QZ (2014) Bone tissue engineering scaffolding: computer-aided scaffolding techniques. Prog Biomater 3:61-102

5. Wang L, Huang Y, Pan K, Jiang X, Liu C (2010) osteogenic responses to different concentrations/ratios of BMP- 2 and bFGF in bone formation. Ann Biomed Eng 38:77-87

6. Su JS, Xu HZ, Sun J, Gong X, Zhao H (2013) Dual delivery of BMP-2 and bFGF from a new nano-composite scaffold, loaded with vascular stents for large-size mandibular defects regeneration. Int J Mol Sci 14:12714-12728

7. Shi Z, Huang X, Cai Y, Tang R, Yang D (2009) Size effect of hydroxyapatite nanoparticles on proliferation and apoptosis of osteoblast-like cells. Acta Biomater 5:338-345

8. Li J, Chen Y, Yin Y, Yao F, Yao K (2007) Modulation of nano-hydroxyapatite size via formation on chitosan-gelatin network film in situ. Biomaterials 28:781-790

9. Rajkumar M, Kavitha K, Prabhu M, Meenakshisundaram N, Rajendran V (2013) Nanohydroxyapatite-chitosan-gelatin polyelectrolyte complex with enhanced mechanical and bioactivity. Mater Sci Eng C 33:3237-3244

10. Thein-Han WW, Misra RD (2009) Biomimetic chitosan-nanohydroxyapatite composite scaffolds for bone tissue engineering. Acta Biomater 5:1182-1197

11. Ji J, Tong X, Huang XF, Zhang JF, Qin HY, Hu QG (2016) Patient-derived human induced pluripotent stem cells from gingival fibroblasts composited with defined nanohydroxyapatite/chitosan/gelatin porous scaffolds as potential bone graft substitutes. Stem Cells Transl Med 5:95-105

12. Van den Dolder J, Jansen JA (2007) The response of osteoblast-like cells towards collagen type I coating immobilized by p-nitrophenylchloroformate to titanium. J Biomed Mater Res A 83:712-719

13. Vandrovcova M, Douglas T, Hauk D, Grössner-Schreiber B, Wiltfang J, Bačáková L et al (2011) Influence of collagen and chondroitin sulfate (CS) coatings on poly-(lactide-co-glycolide) (PLGA) on MG 63 osteoblast-like cells. Physiol Res 60:797-813

14. Wang T, Yang XY, Qi X, Jiang CY (2015) Osteoinduction and proliferation of bone-marrow stromal cells in three-dimensional poly ( $($-caprolactone)/ hydroxyapatite/collagen scaffolds. J Transl Med. 13:152

15. Kawase T, Yamanaka K, Suda Y, Kaneko T, Okuda K, Kogami H et al (2010) Collagen-coated poly (L-lactide-co-varepsilon-caprolactone) film: a promising scaffold for cultured periosteal sheets. J Periodontol 81:1653-1662 
16. Lee TT, Green BA, Dietrich WD, Yezierski RP (1999) Neuroprotective effects of basic fibroblast growth factor following spinal cord contusion injury in the rat. J Neurotrauma 16:347-356

17. Beenken A, Mohammadi M (2009) The FGF family: biology, pathophysiology and therapy. Nat Rev Drug Discov 8:235-253

18. Xu HL, Tian FR, Lu CT, Xu J, Fan ZL, Yang JJ, Chen PP et al (2016) Thermosensitive hydrogels combined with decellularised matrix deliver bFGF for the functional recovery of rats after a spinal cord injury. Sci Rep 6:38332

19. Vanhatupa S, Ojansivu M, Autio R, Juntunen M, Miettinen S (2015) Bone morphogenetic protein-2 induces donor-dependent osteogenic and adipogenic differentiation in human adipose stem cells. Stem Cells Transl Med. 4(12):1391-1402

20. Wang YK, Yu X, Cohen DM, Wozniak MA, Yang MT, Gao L et al (2012) Bone morphogenetic protein-2-induced signaling and osteogenesis is regulated by cell shape, RhoA/ROCK, and cytoskeletal tension. Stem Cells Dev 21(7):1176-1186

21. Castro-Govea Y, Cervantes-Kardasch VH, Borrego-Soto G, Martinez-Rodriguez HG, Espinoza-Juarez M, Romero-Diaz V et al (2012) Human bone morphogenetic protein 2-transduced mesenchymal stem cells improve bone regeneration in a model of mandible distraction surgery. J Craniofac Surg 23:392-396

22. Samorezova JE, Alsberga E (2015) Spatial regulation of controlled bioactive factor delivery for bone tissue engineering. Adv Drug Deliv Rev 84:45-67

23. Song K, Huang M, Shi Q, Du T, Cao Y (2014) Cultivation and identification of rat bone marrow-derived mesenchymal stem cells. Mol Med Rep 10(2):755-760

24. Tong S, Xue L, Xu DP, Liu ZM, Du Y, Wang XK (2016) In vitro culture of hFOB1.19 osteoblast cells on TGF-B1-SF-CS three dimensional scaffolds. Mol Med Rep 13:181-187
25. Wang Z, Lin M, Xie Q, Sun H, Huang Y, Zhang D et al (2016) Electrospun silk fibroin/poly (lactide-co- $\varepsilon$-caprolactone) nanofibrous scaffolds for bone regeneration. Int J NanoMedicine 11:1483-1500

26. Zhang H, Ma X, Zhang L, Guan X, Bai T, Xue C (2015) The ability to form cartilage of NPMSC and BMSC in SD rats. Int J Clin Exp Med 8:4989-4996

27. Gao P, Zhang H, Liu Y, Fan B, Li X, Xiao X et al (2016) Beta-tricalcium phosphate granules improve osteogenesis in vitro and establish innovative osteo-regenerators for bone tissue engineering in vivo. Sci Rep 6:23367

28. Xu C, Lu W, Bian S, Liang J, Fan Y, Zhang X (2012) Porous collagen scaffold reinforced with surfaced activated PLLA nanoparticles. Scientific World J 2012:695137

29. Lee MJ, Sohn SK, Kim KT, Kim CH, Ahn HB, Rho MS et al (2010) Effect of hydroxyapatite on bone integration in a rabbit tibial defect model. Clin Orthop Surg 2:90-97

30. Xia Y, Zhou P, Cheng X, Xie Y, Liang C, Li C et al (2013) Selective laser sintering fabrication of nano-hydroxyapatite/poly- $\varepsilon$-caprolactone scaffolds for bone tissue engineering applications. Int J Nanomed 8:4197-4213

31. Zeng S, Liu L, Shi Y, Qiu J, Fang W, Rong M et al (2015) Characterization of silk fibroin/chitosan 3D porous scaffold and in vitro cytology. PLoS ONE 10:e0128658

\section{Publisher's Note}

Springer Nature remains neutral with regard to jurisdictional claims in published maps and institutional affiliations.

\section{Submit your manuscript to a SpringerOpen ${ }^{\odot}$ journal and benefit from:}

- Convenient online submission

- Rigorous peer review

- Open access: articles freely available online

- High visibility within the field

- Retaining the copyright to your article

Submit your next manuscript at $\boldsymbol{\nabla}$ springeropen.com 